\title{
Desfechos perinatais em gestantes com síndromes hipertensivas: revisão integrativa
}

Perinatal outcomes in pregnant women with hypertensive syndromes: an integrative review Resultados perinatales en mujeres embarazadas con síndromes hipertensivos: una revisión integradora

\section{Alexandra do Nascimento CassianoI, Ana Beatriz Ferreira Vitorino ${ }^{\text {II }}$, Samara Isabela Maia de Oliveira $^{\mathrm{III}}$, Maria de Lourdes Costa da silva ${ }^{\mathrm{IV}}$, Núbia Maria Lima de Sousav, Nilba de Lima Souza ${ }^{\mathrm{vI}}$}

\begin{abstract}
Resumo: Objetivo: identificar na literatura os desfechos perinatais em gestantes com síndromes hipertensivas. Método: trata-se de uma revisão integrativa com coleta de publicações indexadas na LILACS, PUBMED, SCOPUS e WEB OF SCIENCE, durante janeiro de 2017. Os descritores utilizados foram: hipertensão induzida pela gravidez, assistência perinatal e neonatologia. Resultados: os desfechos perinatais de maior incidência foram: mortalidade perinatal, prematuridade, baixo APGAR no $1^{\text {o }}$ e $5^{\circ}$ minuto de vida, recém-nascidos pequenos para idade gestacional, admissão na unidade intensiva, restrição de crescimento intraútero e parto cesariano. Dentre as patologias investigadas, destacou-se a pré-eclampsia $(80,6 \%)$ e $3 \%$ dos artigos abordaram a pré-eclampsia grave. Conclusões: a realização de investigações que analisem a exposição do feto/neonato à condição materna da préeclampsia grave e da hipertensão crônica sobreposta por pré-eclampsia, constitui-se como gaps de conhecimento.

Descritores: Hipertensão induzida pela gravidez; Assistência perinatal; Neonatologia; Enfermagem; Revisão
\end{abstract}

Abstract: Objective: to identify in the literature the perinatal outcomes in pregnant women with hypertensive syndromes. Method: it is an integrative review with collection of publications indexed in LILACS, PUBMED, SCOPUS and WEB OF SCIENCE, during January 2017. The descriptors used were: pregnancy-induced hypertension, perinatal care and neonatology. Results: the most frequent perinatal outcomes were: perinatal

\footnotetext{
I Enfermeira. Mestra em Enfermagem. Universidade Federal do Rio Grande do Norte, Lagoa Natal, Rio Grande do Norte, Brasil. E-mail: anc_enfa@hotmail.com. ORCID: https://orcid.org/0000-0003-0475-5825

II Enfermeira. Mestra em Enfermagem. Secretaria Municipal de Saúde de Natal, Lagoa Natal Rio Grande do Norte, Brasil. E-mail: beatrizviitorino@gmail.com. ORCID: https://orcid.org/0000-0002-1320-1667

III Enfermeira. Mestra em Enfermagem. University of the People, Pasadena, Califórnia, Estados Unidos da América. E-mail: aramas.maia@gmail.com. ORCID: https://orcid.org/0000-0001-7047-6504

IV Enfermeira. Doutora em Enfermagem. Universidade Federal do Rio Grande do Norte, Lagoa Natal Rio Grande do Norte, Brasil. E-mail: lurdinhafoc@hotmail.com. ORCID: https://orcid.org/0000-0001-7251-8966

V Enfermeira. Mestra em Enfermagem. Universidade Federal do Rio Grande do Norte, Lagoa Natal Rio Grande do Norte, Brasil. E-mail: nubiamlsousa@hotmail.com. E-mail: ORCID: https://orcid.org/0000-0001-7724-5661

VI Enfermeira. Doutora em Enfermagem. Universidade Federal do Rio Grande do Norte, Lagoa Natal, Rio Grande do Norte, Brasil. E-mail: nilba.lima@ hotmail.com. ORCID: https://orcid.org/0000-0002-3748-370X
} 
Desfechos perinatais em gestantes com síndromes hipertensivas: Uma revisão integrativa | 2

mortality, prematurity, low APGAR in the 1st and 5th minute of life, newborns small for gestational age, admission to the intensive care unit, intrauterine growth restriction and cesarean delivery. Among the pathologies investigated, pre-eclampsia stood out (80.6\%) and 3\% of the articles addressed severe pre-eclampsia. Conclusions: the realization of investigations that analyze the exposure of the fetus / neonate to the maternal condition of severe pre-eclampsia and chronic hypertension overlapped by pre-eclampsia, constitutes knowledge gaps.

Descriptors: Hypertension, Pregnancy-Induced; Perinatal Care; Neonatology; Nursing; Review

Resumen: Objetivo: identificar en la literatura los resultados perinatales en mujeres embarazadas con síndromes hipertensivos. Método: es una revisión integradora con una colección de publicaciones indexadas en LILACS $^{\circledR}$ PUBMED, SCOPUS y WEB OF SCIENCE, durante enero de 2017. Los descriptores utilizados fueron: hipertensión inducida por el embarazo, atención perinatal y neonatología. Resultados: los resultados perinatales más frecuentes fueron: mortalidad perinatal, prematuridad, APGAR bajo en el primer y quinto minuto de vida, recién nacidos pequeños para la edad gestacional, ingreso a la unidad de cuidados intensivos, restricción del crecimiento intrauterino y parto por cesárea. Entre las patologías investigadas, se destacó la preeclampsia (80,6\%) y el 3\% de los artículos abordó la preeclampsia grave. Conclusiones: la realización de investigaciones que analizan la exposición del feto / recién nacido a la condición materna de preeclampsia severa e hipertensión crónica superpuesta por preeclampsia, se constituye como lagunas de conocimiento.

Descriptores: Hipertensión Inducida en el Embarazo; Atención Perinatal; Neonatología; Enfermería; Revisión

\section{Introdução}

De acordo com o guidelines da American College of Obstetrians and Gynecologists (ACOG), as Síndromes Hipertensivas na Gestação (SHG) são classificadas de acordo com a época de surgimento, entre a $20^{\mathrm{a}}$ semana de gestação e $12^{\mathrm{a}}$ semana do pós-parto, além da presença da proteinúria e dos sinais de gravidade. Com base nisso, suas tipologias se dividem em: Hipertensão Arterial Crônica (HAC), Hipertensão Crônica com Pré-eclampsia Sobreposta (HCPES), Préeclâmpsia isolada (PE), Pré-eclampsia com sinais de gravidade (PEG) e a eclampsia. ${ }^{1}$

A hipertensão durante a gestação é a primeira causa de mortalidade materna no mundo, ocorrendo em 6 a 17\% das gestantes nulíparas e em 2 a $4 \%$ das multíparas, das quais, 20 a $50 \%$ progridem para a PE. ${ }^{2}$ No Brasil, em 2014, a mortalidade materna pelas SHG foi representada pelo coeficiente de 10,8 mortes a cada 100 mil nascimentos. ${ }^{3}$ Paralelo aos dados de mortalidade materna, a taxa de mortalidade neonatal precoce por causas evitáveis, dentre as quais incluem os casos referentes à hipertensão durante a gestação, foi de 2,7 óbitos para uma proporção de 100 mil nascidos vivos, no ano de $2014 .{ }^{4}$ Observa-se que a prematuridade se destaca como umas as principais causas de óbitos 
3 | Cassiano AN, Vitorino ABF, Oliveira SIM, Silva MLC, Sousa NML, Souza NL

neonatais, ${ }^{5}$ sendo estes desfechos recorrentes nos casos de mulheres que desenvolvem as SHG. ${ }^{6}$

Estudo realizado com gestantes que apresentavam diagnóstico de síndromes hipertensivas possuíam risco elevado de terem um recém-nascido (RN) com índice de APGAR abaixo de sete no primeiro $(R R=2,33, p<0,001)$ e quinto minuto $(R R=2,96, p=0,003$, caracterizando-se como hipóxia fetal; além de maior risco relativo para prematuridade $(\mathrm{R} R=2,06, \mathrm{p}=0,017$, baixo peso ao nascer $(R R=2,33, p=0,009$, morte fetal $(R R=2,36, p=0,03)$ e parto cesáreo com desfecho desfavorável $(\mathrm{RR}=4,41, \mathrm{p}<0,001)^{7}$

Os dados chamam atenção enquanto problema de saúde pública, principalmente, por se tratar de complicações preveníveis por meio de uma adequada atenção durante o pré-natal e parto, com ações de promoção à saúde que visem à prevenção das SHG, detecção precoce e acompanhamento da saúde materno-fetal. O conhecimento e discussão sobre as pesquisas realizadas acerca da temática, especialmente, no que diz respeito à identificação de elementos como: país de origem, delineamento, nível de evidência produzida, e os desfechos perinatais investigadas, torna-se relevante para aprofundar a base teórica sobre o assunto e para contribuir com a identificação de gaps de conhecimento. Com isso, torna-se possível instigar a elaboração de novos estudos que preencham as lacunas existentes afim de produzir evidências que subsidiem a tomada de decisões clínicas e que contribuam com o avanço da ciência.

Diante do exposto, o estudo objetiva identificar na literatura os desfechos perinatais em gestantes com síndromes hipertensivas.

\section{Método}

Trata-se de uma revisão integrativa, fundamentada na síntese e análise dos resultados de pesquisas científicas publicadas, a qual segue as seguintes etapas de construção: elaboração do protocolo de revisão integrativa; coleta dos dados com inclusão de publicações que trouxeram contribuições e respostas pertinentes à questão de pesquisa; extração das informações contidas 
Desfechos perinatais em gestantes com síndromes hipertensivas: Uma revisão integrativa $\mid 4$ no estudo; análise e interpretação dos dados; e, por último, apresentação e discussão da revisão. ${ }^{8}$ Para elaboração da questão norteadora, utilizou-se a estratégia PICO conforme recomendação do Reporting Items for Systematic Reviews and Meta-Analyses (PRISMA): Paciente: gestantes com síndromes hipertensivas; Intervenção: não se aplica; Comparação: não se aplica; e Outcomes (desfecho): desfechos perinatais. Com a questão de revisão: quais os desfechos perinatais em gestantes com síndromes hipertensivas evidenciados na literatura?

Foram investigadas as publicações científicas indexadas nas respectivas bases de dados: LILACS, PUBMED, SCOPUS e WEB OF SCIENCE. Cada base de dados foi acessada por dois pesquisadores simultaneamente, em computadores diferentes, com o intuito de garantir fidedignidade ao estudo e coletar o maior número de artigos pertinentes. Não foram adicionados filtros de busca como ano de publicação, país ou revista.

A coleta foi realizada em janeiro de 2017, com uso de descritores selecionados nos Descritores em Ciências da Saúde (DECs) e no Medical Subject Headings (MESH): 1\#Hipertensão induzida pela gravidez/Hypertension, Pregnancy-Induced; 2\#Assistência perinatal/Perinatal Care; 3\# Neonatologia/Neonatology. Os cruzamentos utilizados na busca não controlada foram: (1\# AND 2\#); (1\# AND 3\#) e (1\# AND 2\# AND 3\#). Foram incluídos os artigos completos disponíveis nas bases de dados selecionadas; artigos que evidenciem os desfechos perinatais em gestantes com síndromes hipertensivas. Foram excluídas as publicações em formato de editoriais, cartas ao editor, resumos, opinião de especialistas, revisões, capítulos de livro, teses e dissertações ou que não abordassem o tema de pesquisa.

Em relação à definição do nível de evidência do estudo, utilizou-se a classificação de nível de evidência científica, ${ }^{9}$ a qual estabelece a seguinte classificação: nível I (evidências oriundas de revisões sistemáticas ou meta-análise de relevantes ensaios clínicos); nível II (evidências derivadas de pelo menos um ensaio clínico randomizado controlado bem delineado); nível III (ensaios clínicos bem delineados sem randomização); nível IV (estudos de coorte e de caso-controle bem delineados); 
5 | Cassiano AN, Vitorino ABF, Oliveira SIM, Silva MLC, Sousa NML, Souza NL

nível V (revisão sistemática de estudos descritivos e qualitativos); nível VI (evidências derivadas de um único estudo descritivo ou qualitativo); e nível VII (opinião de autoridades ou relatório de comitês de especialistas. Segundo a mesma classificação os níveis I e II são consideradas evidências fortes; níveis III e V como moderadas e os níveis VI e VII são tidas como evidências fracas.

A seleção das publicações foi iniciada por meio da leitura dos títulos e resumos. Posteriormente foram retirados os estudos não disponíveis na íntegra, duplicados e os que possuíam dados insuficientes para responderem à questão de pesquisa, obtendo-se o total de 31 publicações. A figura 01 apresenta o fluxograma da seleção dos artigos.

Figura 01: Fluxograma da seleção dos artigos baseado nas etapas de identificação, triagem, elegibilidade e inclusão. Natal, Rio Grande do Norte, 2017.
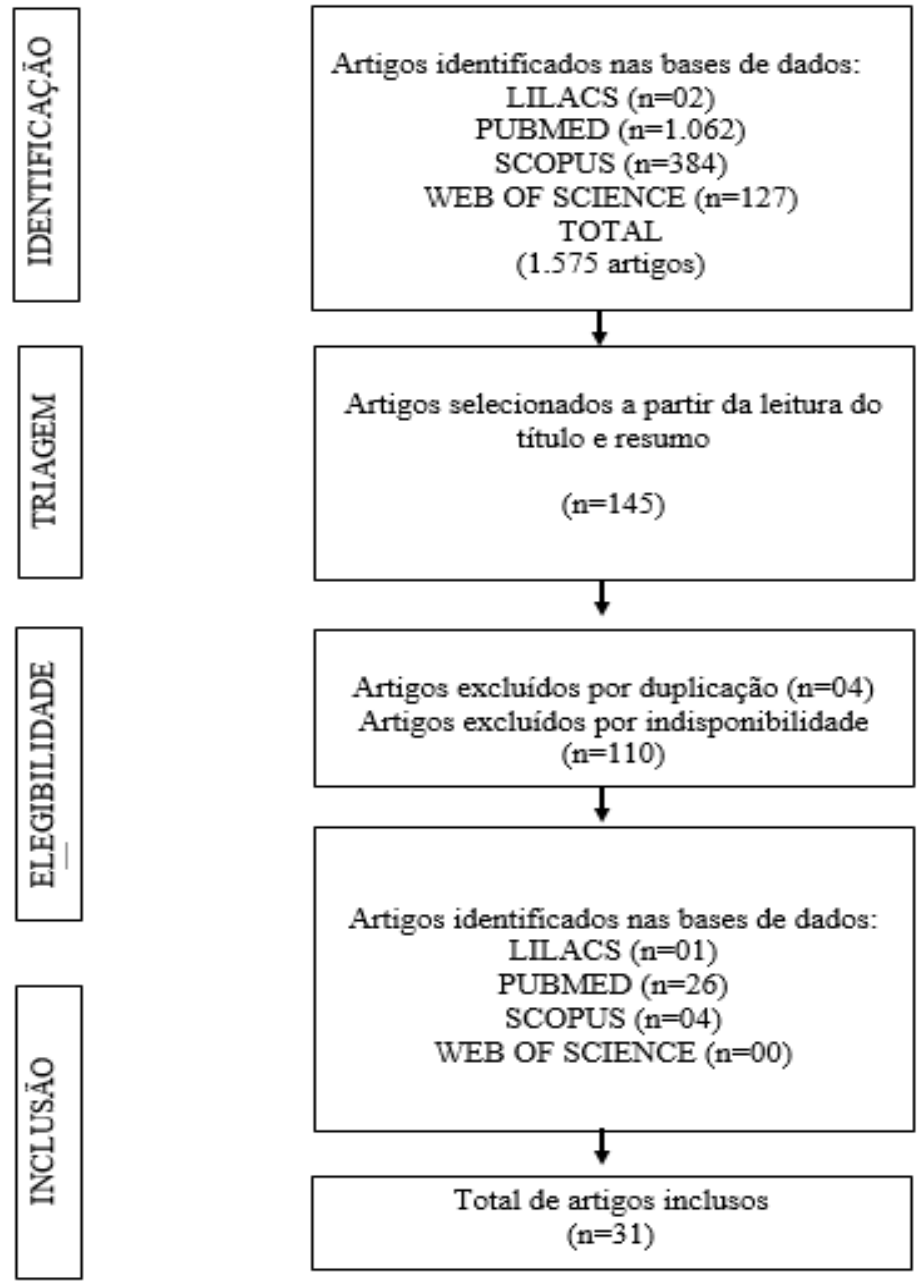

Fonte: Dados coletados pelo autor. 
Desfechos perinatais em gestantes com síndromes hipertensivas: Uma revisão integrativa |6

\section{Resultados}

A caracterização dos artigos quanto as variáveis: revista e ano de publicação, autor, título e os desfechos perinatais identificados nos estudos, estão apresentadas no Quadro 01. As publicações iniciaram em 1998 e seguiram até 2016, com produção média de dois artigos ao ano. Quanto ao idioma, um foi escrito na língua chinesa, dois em português e os demais em inglês. Dos estudos, quatro foram multicêntricos e os demais tiveram os seguintes países de origem: Brasil, Estados Unidos, China, Taiwan, África do Sul, Holanda, Tanzânia, Reino Unido, Paquistão, Austrália, Gana, Singapura, Nigéria, Turquia, Israel, Japão, Argentina, Egito, índia, Peru, África do Sul e Vietnam.

Em relação ao nível de evidência, mais da metade (51\%) foi classificada como nível VI, seguido do IV (45\%) e II (3\%). Dentre os delineamentos adotados, predominou os estudos transversais (51\%), e, em menor número, foram identificados coortes (25\%), casos-controles (19\%) e apenas um ensaio clínico (3\%).

Observou-se que, no Brasil, o quantitativo de pesquisas da temática é limitado (três estudos), dos quais, dois foram desenvolvidos com base em um delineamento transversal e uma coorte. Verificou-se a inexistência de publicações vinculadas à revistas de enfermagem.

Em resposta ao questionamento da presente revisão, obteve-se como resultados que, os desfechos perinatais de maior incidência na vigência das SHG, foram a mortalidade perinatal (A1-2, 4, 6, 8-9, 12-15, 17, 20, 22-30), prematuridade (A4, 6, 8-10, 12-15, 18, 22-25, 27-31) e baixo APGAR no $1^{\circ}$ e $5^{\circ}$ minuto de vida (A2, 4, 6, 8-10, 12, 16-17, 20-22, 27, 29-31). Igualmente, foram verificados desfechos como RN Pequenos para Idade Gestacional (PIG) (A1, 4, 10, 13-23, 25-31), maior ocorrência de admissão na Unidade de Terapia Intensiva Neonatal (UTIN) (A2, 4, 8-9, 15, 18-20, 22, 27), Restrição de Crescimento Intra-uterino (RCIU) (A3, 9, 16-17, 20, 24, 26) e parto cesariano (A4, 14, 16-18, 27, 29, 31).

Seguido aos desfechos citados, também foram identificados resultados como a sepse 
7 | Cassiano AN, Vitorino ABF, Oliveira SIM, Silva MLC, Sousa NML, Souza NL

neonatal (A10, 12, 26), Taquipneia Transitória do RN (TTRN) (A19, 26), hemorragia intraventricular (artigos 10, 19), Síndrome de Aspiração Meconial (SAM) (A3, 10, 21) e Síndrome da Angústia Respiratória (SAR) (A10, 21). Os resultados perinatais identificados com menor frequência foram: hemorragia pulmonar (A21), neutrofilia (A7), aumento do Ácido Tiobarbitúrico (TBARS) (A5), hipotensão neonatal (A11), pneumonia (A26), icterícia (A18), encefalopatia isquêmica (A21), hipoglicemia (A19) e Enterocolite Necrozante (ECN) (A19).

Nos trabalhos, os resultados perinatais em gestantes com diagnóstico de PEG foram citados em 25 pesquisas $(80,6 \%)$, enquanto que em três foram pontuadas a identificação de tais desfechos nos casos que evoluíram com gravidade. Nos demais artigos foram identificados os resultados perinatais em gestantes com HG (12), HAC (11), Eclampsia (08), Síndrome HELLP (04) e HCPES (01). Ressalta-se que em alguns estudos, um mesmo trabalho investigou, concomitantemente, as consequências perinatais em grupos de gestantes com diferentes subtipos de SHG.

Quadro 01: Caracterização dos artigos quanto a identificação, título e os desfechos perinatais identificados nos estudos. Natal, Rio Grande do Norte, Brasil. 2017.

\begin{tabular}{|c|c|c|}
\hline № & $\hat{\mathbf{E}}$ & $\begin{array}{l}\text { DESFECHO } \\
\text { PERINATAL }\end{array}$ \\
\hline A & $\begin{array}{l}\text { Lydakis C, Beevers DG, Beevers M, Lip GYH. Obstrect and } \\
\text { neonatal outcome following chronic hypertension in } \\
\text { pregnancy among differente thinic groups. Q J Med. } \\
\text { 1998;91:847-44. DOI: } 10.1093 / \text { qjimed/91.12.837 }\end{array}$ & $\begin{array}{l}\text { PIG/Baixo peso ao nascer; } \\
\text { Mortalidade neonatal }\end{array}$ \\
\hline A2 & 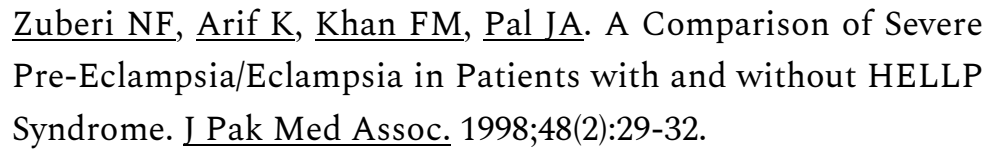 & $\begin{array}{l}\text { Mortalidade perinatal; } \\
\text { PIG; Baixo APGAR; } \\
\text { Admissão na UTIN }\end{array}$ \\
\hline A3 & 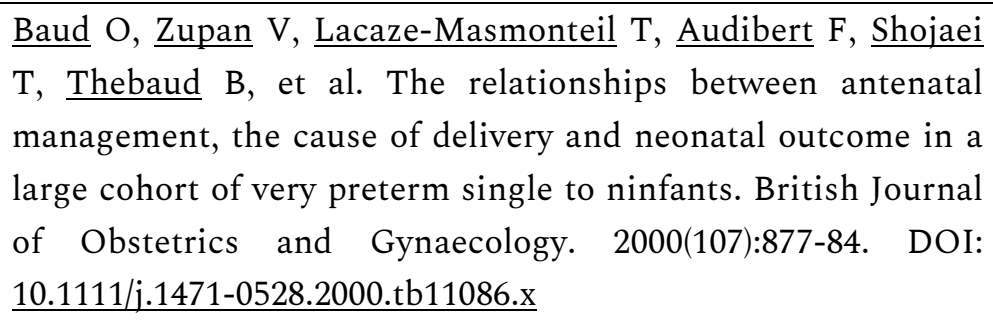 & $\begin{array}{l}\text { RCIU; SAM; Hemorragia } \\
\text { intraventricular grave }\end{array}$ \\
\hline A4 & $\begin{array}{l}\text { Hall DR, Odendaal HJ, Kirsten GF, Smith J, Grové D. } \\
\text { Expectant management of early on set, severe pre-eclampsia: } \\
\text { perinatal outcome. } \quad \text { BJOG. } 2000 ; 107(10): 1258-64 \text { DOI: } \\
\underline{\text { 10.1111/i.1471-0528.2000.tb11617.x }}\end{array}$ & $\begin{array}{l}\text { Cesariana; } \\
\text { Prematuridade; Baixo } \\
\text { APGAR; PIG/Baixo peso } \\
\text { ao nascer; Mortalidade }\end{array}$ \\
\hline
\end{tabular}


Desfechos perinatais em gestantes com síndromes hipertensivas: Uma revisão integrativa $\mid 8$

\begin{tabular}{|c|c|c|}
\hline & & $\begin{array}{l}\text { perinatal; Admissão na } \\
\text { UTIN }\end{array}$ \\
\hline A5 & $\begin{array}{l}\text { Kumari A, Chakrawarty A, Singh A, } \underline{\text { Singh }} \text { R. Urinary } \\
\text { thiobarbituric acid-reacting } \begin{array}{c}\text { substances as potencial } \\
\text { biomarkers of intrauterine hypoxia. I }\end{array} \text { Pregnancy. } \\
\text { 2014;2014:431837. DOI: } 10.1001 / \text { archpedi.155.6.718 }\end{array}$ & $\begin{array}{l}\text { Ácido } \quad \text { tiobarbitúrico } \\
\text { elevado }\end{array}$ \\
\hline A6 & $\begin{array}{l}\text { Shaheen } \mathrm{B} \\
\text { Maternal and Perinatal } \mathrm{L} \text {, Obaid M. Eclampsia, a Major Cause of } \\
\text { tertiary care hospital of Peshawar. L Pak Med Assoc. } \\
\text { 2003;53(8):346-50. }\end{array}$ & $\begin{array}{l}\text { Prematuridade; } \\
\text { Mortalidade perinatal; } \\
\text { Baixo APGAR }\end{array}$ \\
\hline A7 & 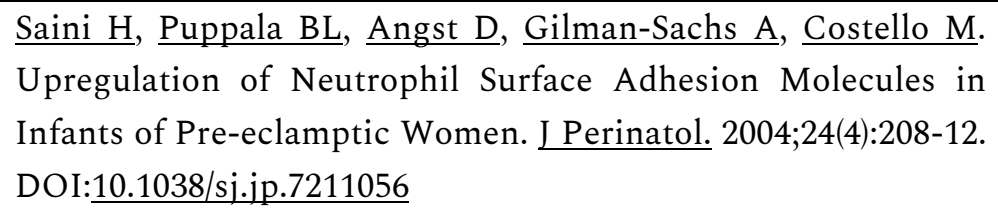 & Neutrofilia \\
\hline A8 & $\begin{array}{l}\text { Roberts CL, Algert CS, Morris JM, Ford JB, } \underline{\text { Henderson-Smart }} \\
\text { DI. Hypertensive disorders in pregnancy: a population-based } \\
\text { study. Med J Aust. 2005;182(7):332-5. DOI: } 10.5694 / \text { j.1326- } \\
\text { 5377.2005.tb06730.x }\end{array}$ & $\begin{array}{l}\text { Prematuridade; } \quad \text { Baixo } \\
\text { APGAR; Admissão na } \\
\text { UTIN; } \quad \text { Mortalidade } \\
\text { perinatal }\end{array}$ \\
\hline A9 & 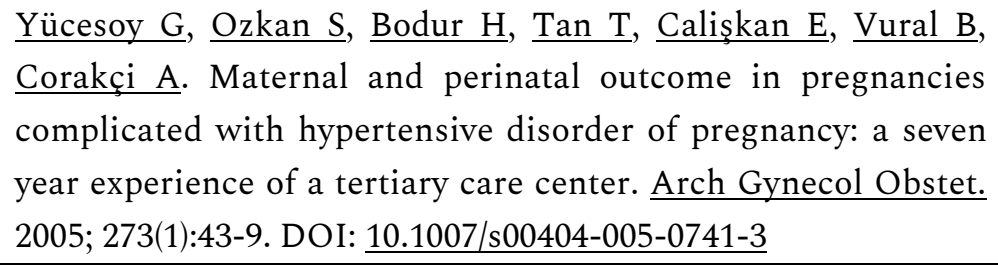 & $\begin{array}{lr}\text { RCIU; Baixo } & \text { APGAR; } \\
\text { Mortalidade } & \text { perinatal; } \\
\text { Prematuridade; } & \text { Admissão } \\
\text { na UTIN } & \end{array}$ \\
\hline A10 & $\begin{array}{c}\text { Oliveira CA, Lins CP, Sá RAM, Netto HC, Bornia RG, Silva } \\
\text { NR, et al. Síndromes hipertensivas da gestação e repercussões } \\
\text { perinatais. Rev. Bras. Saúde Mater. Infant. 2006;6(1):93-98. DOI: } \\
\text { 10.1590/S1519-38292006000100011 }\end{array}$ & $\begin{array}{l}\text { PIG/Baixo peso ao nascer; } \\
\text { Baixo APGAR; Sepse } \\
\text { neonatal; Prematuridade; } \\
\text { SAM; SAR }\end{array}$ \\
\hline A11 & 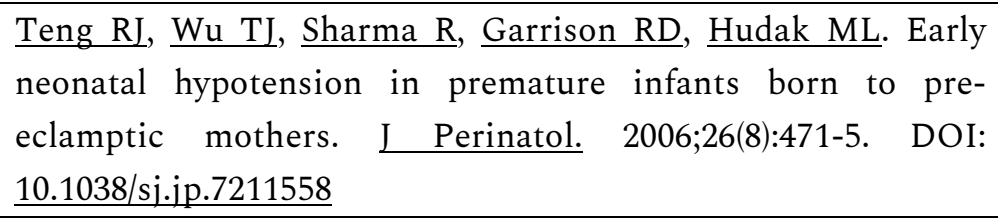 & Hipotensão neonatal \\
\hline A12 & $\begin{array}{l}\text { Ngoc NT, Merialdi M, } \underline{\text { Abdel-Aleem H}}, \underline{\text { Carroli } G}, \underline{\text { Purwar M}} \text {, } \\
\text { Zavaleta N, Campódonico L, et al. Causes of stillbirths and } \\
\text { early neonatal deaths: data from } 7993 \text { pregnancies in six } \\
\text { developing countries. Bull World Health Organ. } 2006 ; 84(9): 699- \\
\text { 705. DOI: } \underline{10.2471 / \text { blt. } 05.027300}\end{array}$ & $\begin{array}{l}\text { Mortalidader perinatal; } \\
\text { Prematuridade; } \quad \text { Baixo } \\
\text { APGAR; Sepse neonatal }\end{array}$ \\
\hline A13 & $\begin{array}{l}\text { Tan KH, Kwek K, Yeo GS. Epidemiology of pre-eclampsia and } \\
\text { eclampsia at the KK Women's and Children's Hospital, } \\
\text { Singapore. Singapore Med J. 2006;47(1):48-53. }\end{array}$ & $\begin{array}{l}\text { Prematuridade; } \\
\text { Mortalitadade perinatal; } \\
\text { PIG/Baixo peso ao nascer }\end{array}$ \\
\hline A14 & $\begin{array}{l}\text { Obed AS, Aniteiye, P. Pregnancy Following Eclampsia: A } \\
\text { Longitudinal Study at Korle Bu Teaching Hospital. Ghana Med } \\
\text { I. 2007;41(3):139-143. }\end{array}$ & $\begin{array}{l}\text { Cesariana; Mortalidade } \\
\text { perinatal; Prematuridade }\end{array}$ \\
\hline
\end{tabular}




\begin{tabular}{|c|c|c|}
\hline & $\begin{array}{l}\text { Vilas FCM, Lúcia TC. Hipertensão e gravidez crônicas } \\
\text { resultados em pacientes com crianças do sexo feminino e seus } \\
\text { fatores que influenciam. Arq. Bras. Cardiol. 2009;93(6 Suppl } \\
\text { 1):159-165. DOI: } 10.1590 / \text { S0066-782X2009001300017 }\end{array}$ & $\begin{array}{l}\text { Prematuridade; PIG/Baixo } \\
\text { peso ao nascer; } \\
\text { Mortalidade perinatal; } \\
\text { Admissão na UTIN }\end{array}$ \\
\hline & $\begin{array}{l}\text { Grisaru-Granovsky } S, \text { Halevy } T, \text { Eidelman } A, \text { Elstein } \mathrm{D} \\
\text { Samueloff A. Hipertensyve disordersog pregnancy and the } \\
\text { small for gestational age neonate: not a simple relationship. } \\
\text { Am J Obstet Gynecol. } 2007 ; 196(4): 335 . e 1-5 \text {. DOI: } \\
\underline{10.1016 / \text { j.ajog.2006.11.003 }}\end{array}$ & $\begin{array}{l}\text { RCIU; Cesariana; Baixo } \\
\text { APGAR; PIG/Baixo peso } \\
\text { ao nascer }\end{array}$ \\
\hline A17 & $\begin{array}{l}\text { Liu CM, Cheng PJ, Chang SD. Maternal Complications and } \\
\text { Perinatal Outcomes Associated with Gestational Hypertension } \\
\text { and Severe Preeclampsia in Taiwanese Women. L Formos Med } \\
\text { Assoc. } 2008 ; 107(2): 129-38 . \text { DOI: } 10.1016 / \text { S0929-6646(08)60126-6. }\end{array}$ & $\begin{array}{l}\text { RCIU; Cesariana; } \\
\text { PIG/Baixo peso ao nascer; } \\
\text { Prematuridade; } \quad \text { Baixo } \\
\text { APGAR; OFIU }\end{array}$ \\
\hline A & $\begin{array}{l}\text { Barton JR, Barton LA, Istwan NB, Desch CN, } \underline{\text { Rhea }} \text { DJ, } \\
\text { Stanziano GJ et al. Elective delivery at } 340 / 7 \text { to } 366 / 7 \text { weeks } \\
\text { gestation and its impacton neonatal outcomes in women with } \\
\text { stable mild gestational hypertension. Am J Obstet Gynecol. } \\
\text { 2011;204:44.e1-5. DOI: https://doi.org/10.1016/j.ajog.2010.08.030 }\end{array}$ & $\begin{array}{l}\text { Prematuridade; PIG/Baixo } \\
\text { peso ao nascer; Admissão } \\
\text { na UTIN; Icterícia }\end{array}$ \\
\hline A19 & $\begin{array}{l}\text { Langenveld J, Ravelli AC, van Kaam AH, van der Ham DP, van } \\
\text { Pampus MG, Porath M. et al. Neonatal outcome of pregnancies } \\
\text { complicated by hypertensive disorders between } 34 \text { and } 37 \\
\text { weeks of gestation: a } 7 \text { year retrospective analysis of a national } \\
\text { registry. Am J Obstet Gynecol. 2011;205(6):540.e1-7. DOI: } \\
\text { 10.1016/j.ajog.2011.07.003. }\end{array}$ & 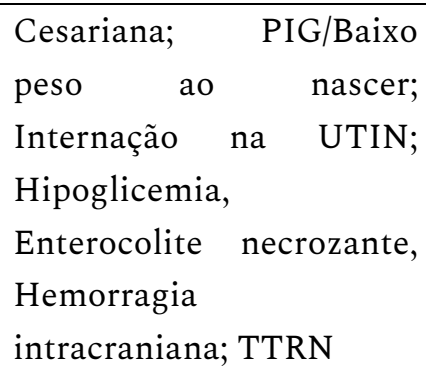 \\
\hline A20 & $\begin{array}{l}\text { Cruz MO, Gao W, Hibbard JU. Obstetrical and perinatal } \\
\text { outcomes among women with gestational hypertensive, mild } \\
\text { preeclampsia, and mild chonic hypertension. Am J Obstet } \\
\underline{\text { Gynecol. } 2011 ; 205(3): 260 . e 1-9 . \text { DOI: } \underline{10.1016 / j . a j o g .2011 .06 .033}}\end{array}$ & $\begin{array}{l}\text { Baixo APGAR; PIG/Baixo } \\
\text { peso ao nascer; RCIU; } \\
\text { Mortalidade neonatal; } \\
\text { Admissão na UTIN }\end{array}$ \\
\hline A21 & $\begin{array}{l}\text { Wei K, Yang Y, Yao Y, Du L, Wang Q, Wang R, Wang L, } \\
\text { Epidemiologic survey on hospitalized neonates in China. } \\
\underline{\text { Transl Pediatr. } 2012 ; 1(1): 15-22 . \text { DOI: } 10.3978 / j . i s s n .2224-} \\
\underline{4336.2011 .10 .01}\end{array}$ & $\begin{array}{l}\text { Baixo APGAR; } \\
\text { Hemorragia pulmonar; } \\
\text { PIG/Baixo peso ao nascer; } \\
\text { SAM; TTRN; SAM; } \\
\text { Encefalopatia isquêmica }\end{array}$ \\
\hline A22 & 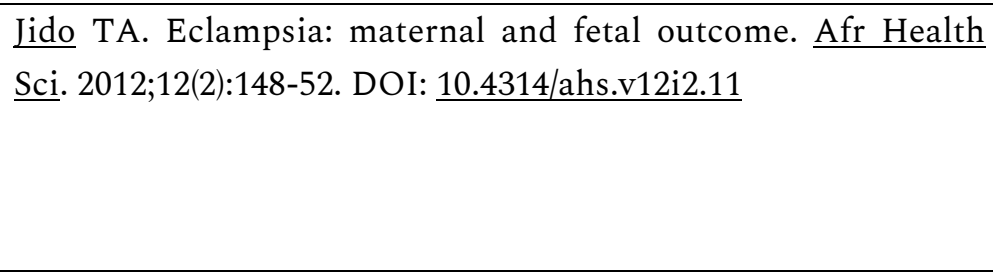 & $\begin{array}{l}\text { Prematuridade; PIG/Baixo } \\
\text { peso ao nascer; Baixo } \\
\text { APGAR; } \\
\text { UTINissão na } \\
\text { perinatal }\end{array}$ \\
\hline A23 & 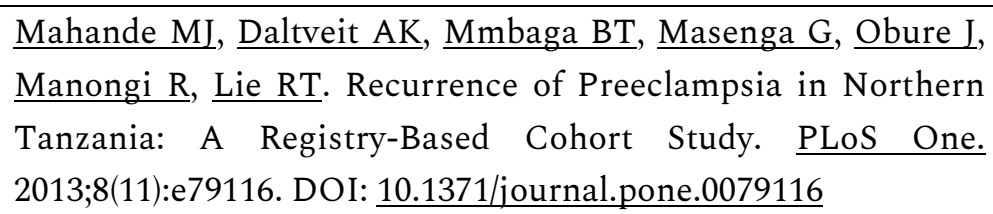 & $\begin{array}{l}\text { Mortalidade perinatal; } \\
\text { PIG/Baixo peso ao nascer; } \\
\text { Prematuridade }\end{array}$ \\
\hline A24 & $\begin{array}{l}\text { Sadaf N, Haq G, Shukar-ud-Din S. Maternal and fetal outcome } \\
\text { in HELLP syndrome at tertiary care hospital. J Pak Med Assoc. }\end{array}$ & $\begin{array}{l}\text { Prematuridade; RCIU; } \\
\text { Mortalidade perinatal }\end{array}$ \\
\hline
\end{tabular}


Desfechos perinatais em gestantes com síndromes hipertensivas: Uma revisão integrativa | 10

\begin{tabular}{|c|c|c|}
\hline & $13 ; 63(12): 1500-3$ & \\
\hline A25 & $\begin{array}{l}\text { Ono Y, Takagi K, Seki H, Takai Y, }, \underline{\text { Samejima K, }}, \underline{\text { Matsunaga S }} \text {, } \\
\text { Matsumura H. Neonatal outcome in infants of chronically } \\
\text { hypertensive mothers. L Obstet Gynaecol Res. } 2013 ; 39(6): 1142- \\
\text { 6. DOI:10.1111/jog. } 12041\end{array}$ & $\begin{array}{l}\text { Prematuridade; PIG/Baixo } \\
\text { peso ao nascer; RCIU; } \\
\text { Mortalidade perinatal }\end{array}$ \\
\hline A26 & $\begin{array}{l}\text { Zanette E, Parpinelli MA, Surita FG, Costa ML, Haddad SM, } \\
\underline{\text { Sousa MH}} \text {, et al. Maternal near miss and death among women } \\
\text { whith severe hypertensive disorders: a Brazilian multicenter } \\
\text { surveil lance study. Reprod Health. 2014;16;11(1):4. DOI: } \\
\underline{10.1186 / 1742-4755-11-4}\end{array}$ & $\begin{array}{lr}\text { Mortalidade } & \text { neonatal; } \\
\text { Pneumonia, } & \text { Sepse } \\
\text { neonatal; RCIU; TTRN }\end{array}$ \\
\hline A27 & $\begin{array}{l}\text { Abalos E, Cuesta C, Carroli G, Qureshi Z, Widmer M, Vogel JP } \\
\text { et al. Pre-eclampsia, eclampsia and adverse maternal and } \\
\text { perinatal outcomes: a secondary analysis of the World Health } \\
\text { Organization Multicountry Survey on Maternal and Newborn } \\
\text { Health. BJOG. 2014;121 Suppl 1:14-24. DOI: } \underline{10.1111 / 1471-} \\
\underline{0528.12629}\end{array}$ & $\begin{array}{l}\text { Cesariana; } \\
\text { Prematuridade; PIG/Baixo } \\
\text { peso ao nascer; } \\
\text { Mortalidade perinatal; } \\
\text { Baixo APGAR; } \\
\text { na UTINissão }\end{array}$ \\
\hline A28 & 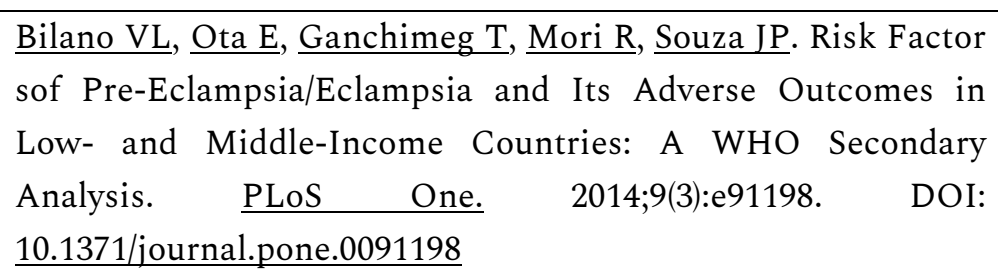 & $\begin{array}{l}\text { Mortalidade perinatal; } \\
\text { Prematuridade; PIG/Baixo } \\
\text { peso ao nascer }\end{array}$ \\
\hline A29 & $\begin{array}{l}\text { Vogel JP, Souza JP, Mori } \mathrm{R}, \text { Morisaki } \mathrm{N} \text {, Lumbiganon } \mathrm{P} \\
\text { Laopaiboon } \mathrm{M} \text {, et al. Maternal complications and perinatal } \\
\text { mortality: findings of the World Health Organization } \\
\text { Multicountry Survey on Maternal and Newborn Health. BJOG. } \\
\text { 2014;121 Suppl 1:76-88. DOI: } 10.1111 / 1471-0528.12633\end{array}$ & $\begin{array}{l}\text { Prematuridade PIG/Baixo } \\
\text { peso ao nascer; Baixo } \\
\text { APGAR; Mortalidade } \\
\text { perinatal; Cesareana }\end{array}$ \\
\hline A30 & $\begin{array}{l}\text { Browne JL, Vissers KM, } \\
\text { Linden EL }, \underline{\text { Antwi E }}, \underline{\text { Srofenyoh EK, }}, \underline{\text { Van der }} \\
\text { hypertensive disorders in pregnancy in a low resource setting. } \\
\text { Trop Med Int Health. } 2015 ; 20(12): 1778-86 . \quad \text { DOI: } \\
10.1111 / \text { tmi.12606 }\end{array}$ & $\begin{array}{l}\text { Prematuridade; PIG/Baixo } \\
\text { peso ao nascer; Baixo } \\
\text { APGAR; } \quad \text { Mortalidade } \\
\text { perinatal }\end{array}$ \\
\hline A31 & $\begin{array}{l}\text { Oliveira ACM, Santos AA, Bezerra AR, Barros AMR, Tavares } \\
\text { MCM. Fatores Maternos e Resultados Perinatais Adversos em } \\
\text { Portadoras de Pré-eclâmpsia em Maceió, Alagoas. Arq Bras } \\
\text { Cardiol. 2016. DOI: } 10.5935 / \text { abc. } 20150150\end{array}$ & $\begin{array}{l}\text { Prematuridade; PIG/Baixo } \\
\text { peso ao nascer; Baixo } \\
\text { APGAR; Cesariana }\end{array}$ \\
\hline
\end{tabular}

${ }^{*}$ A: Artigo

Fonte: Dados coletados pelo autor.

\section{Discussão}


11 | Cassiano AN, Vitorino ABF, Oliveira SIM, Silva MLC, Sousa NML, Souza NL

Embora a publicação de pesquisas sobre o tema tenha sido contínua nos últimos 15 anos e em diferentes países, o conhecimento acerca dos desfechos perinatais na vigência das SHG, especialmente, no contexto brasileiro, permanece enquanto uma área ainda pouco explorada pelas ciências, incluindo a enfermagem. Há, portanto, a necessidade de fomentar o desenvolvimento de estudos que abordem tal assunto, haja vista a significativa incidência da hipertensão em gestantes e a gravidade da mesma para mãe e o feto/neonato. ${ }^{10}$

Nessa revisão, a mortalidade perinatal foi identificada como o resultado negativo de maior ocorrência nas mulheres diagnosticadas com algum tipo de síndrome hipertensiva, que pode ser verificada nos estudos A1-2, 4, 6, 8-9, 12-15, 17, 20 e 22-30. No Brasil, as afecções originadas no período perinatal, dentre as quais se incluem aquelas decorrentes dos transtornos hipertensivos maternos, despontam como a principal causa de mortalidade durante o período, ${ }^{11}$ apesar de essas serem consideradas como condições passíveis de redução por meio uma adequada atenção à mulher na gestação, parto e ao RN. ${ }^{12}$

Atendendo o exposto acima, são consideradas afecções originadas no período perinatal os transtornos respiratórios e cardiovasculares; sepse neonatal; desordens relacionadas à duração da gestação e ao crescimento fetal, a exemplo do baixo peso ao nascer e a prematuridade; bem como as complicações maternas que interfiram na evolução habitual da gravidez, trabalho de parto e parto. ${ }^{11,13}$ Desfechos esses recorrentes na evolução da gravidez de mulheres com hipertensão.

No contexto do Sistema Único de Saúde (SUS) são estimadas como causas evitáveis de óbito perinatal, aquelas que seriam preveníveis pelo acesso e garantia de uma assistência qualificada dos serviços de saúde durante o ciclo gravídico-puerperal. ${ }^{12}$ Portanto, a síntese dos estudos demonstra que a mortalidade perinatal se apresenta enquanto resultado que persistente na realidade, mesmo diante do avanço das tecnologias assistenciais e das políticas públicas de atenção à saúde da mulher.

Especificamente em relação à ocorrência do OFIU, um estudo do tipo caso-controle, 
Desfechos perinatais em gestantes com síndromes hipertensivas: Uma revisão integrativa | 12

realizado no município de Cuiabá, Mato Grosso do Sul (MG), Brasil, de 2006 a 2010, concluiu que os transtorno maternos hipertensivos foram a segunda causa responsável pela mortalidade fetal, antecedida, apenas, pelas causas não especificadas. ${ }^{14}$

Seguida da mortalidade perinatal, a prematuridade (A 4, 6, 8-10, 12-15, 18, 22-25, 27-31) se coloca como a segunda consequência mais recorrente em gestantes hipertensas, corroborando com os achados de outros estudos, nos quais, o nascimento pré-termo esteve significativamente associado a elevação de níveis pressóricos e, mais frequentemente, à pré-eclampsia. ${ }^{7,15-16}$

As condições ao nascimento, representadas pelo Índice de APGAR, parecem ser piores a depender da tipologia da SHG segundo verificado nos artigos A2, 4, 6, 8-10, 12, 16-17, 20-22, 27 e 29-31. Dessa forma, neonatos de mães com eclampsia, apresentam scores baixos de APGAR até no $5^{\circ}$ minuto, ${ }^{17}$ ao passo que, aqueles nascidos de gestantes com HG e PEG também nasceram hipóxicos, porém em menor frequência. ${ }^{15,18}$ Mesmo diante das evidências encontradas, um estudo não identificou diferença quanto ao APGAR entre grupos de RN de mães hipertensas e normotensas, e, a partir de tal resultado, buscou ressaltar que o índice sofre interferência da prematuridade e de anomalias congênitas. $^{6}$

No presente trabalho, $43,7 \%$ dos estudos relataram em seus resultados uma associação entre a elevação dos níveis pressóricos maternos e a presença da PEG com o baixo peso ao nascimento e o tamanho PIG (A 1, 4, 10, 13-23, 25-31) 2,18. Em consequência a tais desfechos adversos a admissão na UTIN (A2, 4, 8-9, 15, 18-20, 22, 27) para cuidados especiais, principalmente em decorrência da asfixia e da prematuridade, é constatada com considerável incidência nos casos de gestantes hipertensas. ${ }^{17-18}$

Notadamente, a tecnologia assistencial das unidades intensivas propiciaram a redução da mortalidade ao permitir a recuperação de RN graves. No entanto, a manipulação por profissionais e a submissão às intervenções acaba por expor o neonato a eventos adversos e riscos prejudiciais à saúde dos mesmos, sobretudo quando os períodos de internação são prolongados. ${ }^{19}$ 
13 | Cassiano AN, Vitorino ABF, Oliveira SIM, Silva MLC, Sousa NML, Souza NL

Apesar da RCIU ser apresentada como o $6^{\circ}$ desfecho mais citado pelas pesquisas, como nos trabalhos A3, 9, 16-17, 20, 24 e 26, é consenso na literatura que sua fisiopatologia tem estreita relação com os transtornos hipertensivos maternos. A PEG, por exemplo, promove insuficiência placentária severa, sujeitando o feto a hipóxia. ${ }^{12}$ Nos casos de HCPES a restrição do crescimento também é um achado comum. ${ }^{20}$

Não obstante, a interrupção da gravidez em gestantes hipertensas por meio de cesariana $(\mathrm{A} 4,14,16-18,27,29,31)$ eletiva persiste enquanto um assunto controverso na literatura, embora sua ocorrência seja significativa nos casos que evoluem com gravidade, a exemplo da PEG. . $^{6,15}$

Segundo conduta protocolada pelo Ministério da Saúde (MS) a resolução da gravidez é recomendada apenas nas situações em que há o agravamento das condições maternas e/ou fetal, devendo, preferencialmente, ser adotada as condutas conservadoras e/ou expectante até a 34 semana de gestação. ${ }^{21}$ Quando considerada as evidências disponíveis para basear a indicação de cesarianas em gestantes hipertensas, uma revisão sistemática apontou os respectivos critérios: “Frequência Cardíaca Fetal (FCF) não tranquilizadora”, “oligodrâmnia” e "centralização Fetal”, os quais são igualmente indicados pelas diretrizes ministeriais, e possuem grau de recomendação $\mathrm{B}^{22}$

A sepse neonatal também correspondeu a uma descoberta associada à neonatos de mães com $\mathrm{HG},{ }^{2}$ identificada nos artigos A10, 12 e 26. Existe na literatura o consenso de que a etiologia da infecção durante esse período é multifatorial, abrangendo fatores relacionados à condições maternas e neonatais, a exemplo do trabalho de parto prematuro, ruptura de membranas com mais de 18 horas, infecção materna, baixo peso ao nascer, prematuridade, ventilação mecânica (VM) com Tudo Orotraqueal (TOT), cateter central ou umbilical e nutrição parenteral. ${ }^{23}$

Estudos identificaram que a vigência materna de HG e HAC aumentou o risco para a ocorrência de disfunções respiratórias como TTRN (A19, 26), SAM (A3, 10, 21), SAR (A10, 21), além da hemorragia e lesão pulmonar (A21) em RN pré-termos e com baixo peso. ${ }^{2,12}$ Observa-se 
Desfechos perinatais em gestantes com síndromes hipertensivas: Uma revisão integrativa | 14

que as intercorrências citadas acima tem estreita relação com a condição de prematuridade, uma vez que complicações dessa natureza são secundárias ao nascimento pré-termo, o qual se constitui como uma das principais complicações das SHG.

Achados neurológicos em RN prematuros de mães hipertensas são controversos e pouco frequentes nas pesquisas, sendo os mais incidentes, a hemorragia intraventricular (A21), a encefalopatia isquêmica e a leucomalácia periventricular. ${ }^{6}$ A frequência de complicações como a ECN (A19), neutrofilia (A7), aumento do TBARS (A5), hipotensão neonatal (A11), pneumonia (A21), icterícia (A18) e hipoglicemia (A19) foram menores, porém as consequências dessas para a saúde neonatal são de significativo impacto.

Posto isso, a ECN acomete de maneira especial prematuros de baixo peso, constituindo-se como a mais comum emergência clínica/cirúrgica e a maior causa de morbimortalidade de RN em UTIN no mundo. ${ }^{24}$ Sua etiologia é desconhecida, mas, possui fatores associados como prematuridade, peso ao nascer, asfixia, sepse, choque, ventilação mecânica, além de evidências que sugerem sua associação com a HG..$^{25}$

Chama atenção alterações bioquímicas como neutrofilia, elevação do TBARS e hipoglicemia. Um caso controle realizado com lactentes de mães com PE e normotensas, revelou a ativação de neutrófilos proporcionalmente à gravidade da doença hipertensiva; ${ }^{26}$ enquanto que, em relação a elevação do TBARS, a detecção do mesmo na urina de RN de mães com HG se constutui enquanto biomarcador que avalia a exposição do feto a hipóxia intrauterina crônica. ${ }^{27}$

A hipoglicemia é comumente verificada em RN macrossômicos e filhos de mães diabéticas, apesar disso, um estudo identificou uma expressiva incidência da hipoglicemia em neonatos de mães com HG ${ }^{28}$ Quanto a hipotensão neonatal, esse é um problema comum nas UTIN, maiormente em RN prematuros, e, mais recentemente, a PE materna foi associada a sua ocorrência. ${ }^{29}$ Por fim, RN prematuros de mães hipertensas apresentam associação com o diagnóstico neonatal de 
15 | Cassiano AN, Vitorino ABF, Oliveira SIM, Silva MLC, Sousa NML, Souza NL

icterícia, ${ }^{30}$ uma vez que, dentre os fatores de risco para hiperbilirrubinemia neonatal, encontra-se um dos desfechos considerados recorrentes nas SHG, a prematuridade.

Destarte, as repercussões das SHG para os desfechos perinatais são de significativo impacto, sejam elas diretas, como no caso da mortalidade perinatal, do parto cesariano e da prematuridade; ou indiretas, a exemplo dos desfechos secundários a prematuridade como o baixo peso ao nascer, admissão na UTIN, sepse e demais citados.

\section{Conclusões}

Os desfechos perinatais mais incidentes na vigência das SHG foram: mortalidade perinatal, prematuridade, baixo APGAR no $1^{\circ}$ e $5^{\circ}$ minuto de vida, RNPIG, maior ocorrência de admissão na UTIN, RCIU e parto cesariano.

Dentre as síndromes hipertensivas investigadas, destacou-se o quantitativo de estudos com gestantes diagnosticadas com $\mathrm{PE}$, porém, verificou-se um menor número de trabalhos com o objetivo analisar os desfechos perinatais em gestantes com PEG e HCPES. Portanto, a realização de investigações que analisem a exposição do feto/neonato à condição materna de ambas síndromes, constitui-se como gaps de conhecimento. Outras tipologias das SHG foram pesquisadas como a HG, HAC, Eclampsia e HELLP.

Tendo em vista o ínfimo número de estudos realizados no Brasil, recomenda-se o desenvolvimento de pesquisas com delineamentos de maior nível de evidência, as quais objetivem investigar as repercussões das SHG no contexto nacional. Isso porque o perfil socioeconômico das mulheres brasileiras é congruente a elementos considerados como fatores de risco para as SHG, a exemplo da primiparidade em mulheres abaixo de 20 anos, cor negra, baixa escolaridade e renda.

A revisão apresenta como limitações o número de bases de dados pesquisadas, a indisponibilidade de artigos não gratuitos e a não utilização de instrumentos para avaliação dos 
Desfechos perinatais em gestantes com síndromes hipertensivas: Uma revisão integrativa | 16

artigos científicos.

\section{Referências}

1. American College of Obstetricians and Gynecologists (ACOG). Hypertension in pregnancy. Obstet Gynecol [Internet]. 2013 [acesso em 2016 out 04];122(5):1122-31. Disponível em: https://www.ncbi.nlm.nih.gov/pubmed/24150027

2. Oliveira CA, Lins CP, Sá RAM, Netto HC, Bornia RG, Silva NR, et al. Síndromes hipertensivas da gestação e repercussões perinatais. Rev Bras Saúde Mater Infant [Internet]. 2006 [acesso em 2016 mar 12];6(1):93-8. Disponível em: http://www.scielo.br/scielo.php?script=sci_arttext\&pid=S151938292006000100011

3. Ministério da Saúde (BR), Departamento de Saúde e Vigilância de Doenças Não Transmissíveis. Painel de monitoramento da mortalidade materna. Óbitos maternos declarados. Causas obstétricas diretas (hipertensão) [Internet]. 2015 [acesso em 2016 jul 04]. Disponível em: http://svs.aids.gov.br/dantps/centrais-de-conteudos/paineis-de-monitoramento/mortalidade/materna/

4. Ministério da Saúde (BR), Departamento de Saúde e Vigilância de Doenças Não Transmissíveis. Painel de monitoramento da mortalidade infantil e fetal. Óbitos de causas evitáveis no período neonatal precoce. Óbitos reduzíveis por adequada atenção à mulher na gestação [Internet]. 2015 [acesso em 2016 jul 04]. Disponível em: http://svs.aids.gov.br/dantps/centrais-de-conteudos/paineis-demonitoramento/mortalidade/infantil-e-fetal/

5. França EB, Lanskyl S, Rego MAS, Malta DC, França JS, Teixeira R, et al. Principais causas da mortalidade na infância no Brasil, em 1990 e 2015: estimativas do estudo de Carga Global de Doença. Rev Bras Epidemiol [Internet]. 2017 [acesso em 2019 out 09];20 Supl 1:46-60. Disponível em: http://www.scielo.br/pdf/rbepid/v20s1/1980-5497-rbepid-20-s1-00046.pdf

6. Moura MDR, Castro MP, Margotto PR, Rugolo LMSP. Hipertensão arterial na gestação: importância do seguimento materno no desfecho neonatal. Comun Ciênc Saúde [Internet]. 2011 [acesso em 2016 jul 04];22 Supl 1:113-20. Disponível em: http://bvsms.saude.gov.br/bvs/artigos/hipertensao_arterial_gestacao.pdf

7. Antunes MB, Demitto MO, Gravena AAF, Padovani C, Pelloso SM. Síndrome hipertensiva e resultados perinatais em gestação de alto risco. REME Rev Min Enferm [Internet]. 2017 [acesso em 2019 out 09];21:1-6. Disponível em: http://www.reme.org.br/artigo/detalhes/1195

8. Botelho LLR, Cunha CCA, Macedo M. O método da revisão integrativa nos estudos organizacionais. Gest Soc [Internet]. 2016 [acesso em 2016 mar 12];5(11):121-36. Disponível em: https://www.gestaoesociedade.org/gestaoesociedade/article/view/1220 
9. Melnyk BM, Fineout-Overholt E. Making the case for evidence-based practice [Internet]. Philadelphia: Lippincot Williams \& Wilkins; 2005 [acesso em 2019 out 09]; Evidence-based practice in nursing \& healthcare. A guide to best practice. p. 3-24. Disponível em: https://www.researchgate.net/publication/281080516_Making_the_case_for_evidencebased_practice_and_cultivating_a_spirit_of_inquiry

10. Martins M, Monticelli M, Brüggemann OM, Costa RA. Produção de conhecimento sobre hipertensão gestacional na pós-graduação stricto sensu da enfermagem brasileira. Rev Esc Enferm USP [Internet]. 2012 [acesso em 2016 jul 04];46(4):802-8. Disponível em: http://www.scielo.br/scielo.php?script=sci_arttext\&pid=S0080-62342012000400003

11. Gaiva MAM, Fujimori E, Sato APS. Mortalidade neonatal: análise das causas evitáveis. Rev Enferm UERJ [Internet]. 2015 [acesso em 2017 jan 12];23(2):247-53. Disponível em: http://www.facenf.uerj.br/v23n2/v23n2a17.pdf

12. Malta DC, Duarte EC, Almeida MF, Dias MAS, Morais Neto OL, Moura L, et al. Lista de causas de mortes evitáveis por intervenções do Sistema Único de Saúde do Brasil. Epidemiol Serv Saúde [Internet]. 2007 [acesso em 2016 jul 04];16:233-44. Disponível em: http://scielo.iec.gov.br/pdf/ess/v16n4/v16n4a02.pdf

13. Moura MDR, Margotto PR, Rugolo LMSS. Alterações do fluxo sanguíneo em artéria umbilical na síndrome hipertensiva gestacional e suas implicações nos resultados neonatais. Rev Bras Ginecol Obstet [Internet]. 2013 [acesso em 2017 jan 12];35(2):71-7. Disponível em: http://www.scielo.br/scielo.php?script=sci_arttext\&pid=S0100-72032013000200006

14. Lima JC, Oliveira Júnior GJ, Takano AO. Fatores associados à ocorrência de óbitos fetais em Cuiabá, Mato Grosso. Rev Bras Saúde Matern Infant [Internet]. 2016 [acesso em 2016 jul 04];16(3): 363-71. Disponível em: http://www.scielo.br/scielo.php?pid=S151938292016000300353\&script=sci_arttext\&tlng=pt

15. Oliveira ACM, Santos AA, Bezerra AR, Barros AMR, Tavares MCM. Fatores maternos e resultados perinatais adversos em portadoras de pré-eclâmpsia em Maceió, Alagoas. Arq Bras Cardiol [Internet]. 2016 [acesso em 2016 jul 04];106(2):113-20. Disponível em: http://www.scielo.br/pdf/abc/2016nahead/pt_0066-782X-abc-20150150.pdf

16. Gonzaga ICA, Santos SLD, Silva ARV, Campelo V. Atenção pré-natal e fatores de risco associados à prematuridade e baixo peso ao nascer em capital do nordeste brasileiro. Ciênc Saúde Colet [Internet]. 2016 [acesso em 2017 jan 12];(6):1965-74. Disponível em: http:/www.scielo.br/scielo.php?pid=S1413$81232016000601965 \&$ script=sci_abstract\&tlng=pt

17. Jido TA. Ecalmpsia: maternal and fetal outcome. Afr Health Sci [Internet]. 2012 [acesso em 2017 jan 12];12(2):148-52. Disponível em: https://www.ncbi.nlm.nih.gov/pmc/articles/PMC3462530/ 
Desfechos perinatais em gestantes com síndromes hipertensivas: Uma revisão integrativa | 18

18. Zanette E, Parpinelle MA, Surita FG, Costa ML, Haddad SM, Souza MH, et al. Maternal near miss and death among women with severe hypertensive disorders: a Brazilian multicenter surveillance study. Reprod Health [Internet]. 2014 [acesso em 2016 jul 04];11(4):1-11. Disponível em: http://reproductivehealth-journal.biomedcentral.com/articles/10.1186/1742-4755-11-4

19. Lanzillotti LS, Seta MH, Andrade CLT, Mendes Junior WV. Eventos adversos e outros incidentes na unidade de terapia intensiva neonatal. Ciênc Saúde Colet [Internet]. 2015 [acesso em 2017 mar 03];20(3):937-46. Disponível em: http://www.scielo.br/pdf/csc/v20n3/pt_1413-8123-csc-20-03-00937.pdf

20. Henrique AJ, Borrozzino NF, Gabrielloni MC, Barbieri M, Schirmer J. Resultado perinatal em mulheres portadoras de hipertensão arterial crônica: revisão integrativa da literatura. Rev Bras Enferm [Internet]. 2012 [acesso em 2017 jan 12];65(6):1000-10. Disponível em: http://www.scielo.br/pdf/reben/v65n6/a17v65n6.pdf

21. Ministério da Saúde (BR), Secretária de Atenção à Saúde, Departamento de Ações Programáticas e Estratégicas. Gestação de Alto Risco: manual técnico. 5ª ed. Brasília (DF): Ministério da Saúde; 2010; 302 p.

22. Amorim MMR, Souza ASR, Porto AMF. Indicações de cesariana baseadas em evidências: parte I. Femina [Internet]. 2010 [acesso em 2017 jan 12];38(8):415-22. Disponível em: http://bhpelopartonormal.pbh.gov.br/estudos_cientificos/arquivos/cesariana_baseada_evidencias_parte_I.pdf

23. Cunha RCML, Araújo GC, Borges MRMM, Queiroz MVF, Pimenta RS. Prevalência de sepse e fatores de risco em neonatos de unidade de terapia intensiva de referência em Palmas, Tocantins, Brasil. Rev Panam Infectol [Internet]. 2014 [acesso em 2017 mar 03];16(2):86-94. Disponível em: https://docplayer.com.br/33067560-Prevalencia-de-sepse-e-fatores-de-risco-em-neonatos-de-unidade-deterapia-intensiva-de-referencia-em-palmas-tocantins-brasil.html

24. Chu A, Hageman JR, Caplan MS. Necrotizing enterocolitis: Predictive markers and preventive strategies. Neoreviews [Internet]. 2013 [acesso em 2017 jan 12];14(3):113-20. Disponível em: https://www.researchgate.net/publication/275622619_Necrotizing_Enterocolitis_Predictive_Markers_and _Preventive_Strategies

25. Vieira AA, David BBL, Lino RRG, Duarte LB, Bueno AC. Avaliação dos fatores perinatais que interferem na incidência de enterocolite necrosante em recém-nascidos de muito baixo peso. Rev Bras Ginecol Obstet [Internet]. 2013 [acesso em 2017 jan 12];35(8):363-7. Disponível em: http://www.scielo.br/scielo.php?script=sci_abstract\&pid=S0100-72032013000800005\&lng=e\&tlng=pt

26. Saini H, Puppala BL, Angst D, Gilman-Sachs A, Costello M. Upregulation of neutrophil surface adhesion molecules in infants of pre-eclamptic women. J Perinatol [Internet]. 2004 [acesso em 2017 jan 12];24:208-12. Disponível em: http://www.nature.com/jp/journal/v24/n4/full/7211056a.html?message=removeacesso 
19 | Cassiano AN, Vitorino ABF, Oliveira SIM, Silva MLC, Sousa NML, Souza NL

27. Siciarz A, Weinberger B, Witz G, Hiatt M, Hegyi T. Urinary thiobarbituric acid-reacting substances as potencial biomarkers of intrauterine hypoxia. Arch Pediatr Adolesc Med [Internet]. 2011 [ acesso em

2017 jan 12];155:718-22. Disponível em: http://jamanetwork.com/journals/jamapediatrics/fullarticle/190721

28. Freitas P, Matos CV, Kimura AF. Perfil das mães de neonatos com controle glicêmico nas primeiras horas de vida. Rev Esc Enferm USP [Internet]. 2010 acesso em 2017 fev 03];44(3):636-41. Disponível em: http://www.scielo.br/scielo.php?script=sci_arttext\&pid=S0080-62342010000300012

29. Teng R, Wu TJ, Sharma R, Garrison RD, Hudak ML. Early neonatal hypotension in premature infants born to preeclamptic mothers. J Perinatol [Internet]. 2006 [acesso em 2017 jan 12];26:471-5. Disponível em: https://www.ncbi.nlm.nih.gov/pubmed/16775620

30. Barton JR, Barton LA, Istwan NB, Desch CN, Rhea DJ, Stanziano GJ, et al. Elective delivery at 37 to 36 weeks gestation and its impacto on neonatal outcomes in women with stable mild gestational hypertension. Am J Obstet Gynecol [Internet]. 2011 [acesso em 2016 jul 04];204(44):1-5. Disponível em: http://www.ajog.org/article/S0002-9378(10)01033-1/abstract

\section{Autor correspondente}

Alexandra do Nascimento Cassiano

E-mail: anc_enfa@hotmail.com

Endreço: Campus Universitário, Lagoa Nova, Natal, Rio Grande do Norte, Brasil

CEP: 59. 078-970

\section{Contribuições de Autoria}

\section{1 - Alexandra do Nascimento Cassiano}

Concepção e planejamento, obtenção e análise dos dados, redação e revisão crítica.

\section{2 - Ana Beatriz Ferreira Vitorino}

Obtenção dos dados e revisão crítica.

3 - Samara Isabela Maia de Oliveira

Obtenção dos dados e revisão crítica.

\section{4- Maria de Lourdes Costa da silva}

Planejamento, obtenção dos dados e revisão crítica.

5- Núbia Maria Lima de Sousa

Obtenção dos dados e revisão crítica.

6- Nilba de Lima Souza 
Desfechos perinatais em gestantes com síndromes hipertensivas: Uma revisão integrativa | 20

Concepção, planejamento e revisão crítica.

\section{Como citar este artigo}

Cassiano AN, Vitorino ABF, Oliveira SIM, Silva MLC, Sousa NML, Souza NL. Desfechos perinatais em gestantes com síndromes hipertensivas: Uma revisão integrativa. Rev. Enferm. UFSM. 2020 [Acesso em: Anos Mês Dia]; vol.10 e23: 1-20. DOI:https://doi.org/10.5902/2179769233476 